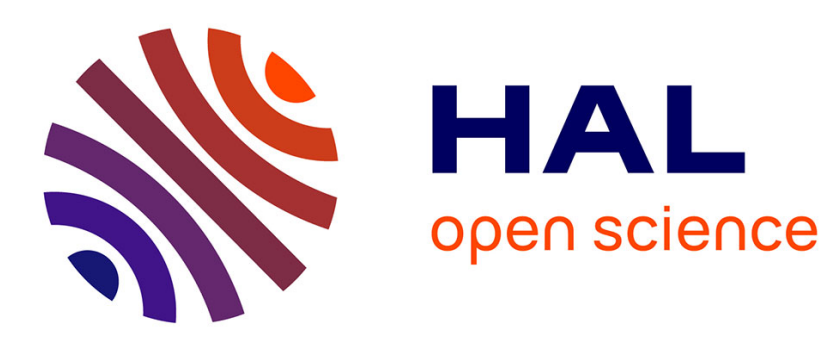

\title{
Ségrégation intergranulaire du bore dans Fe-40 at\% Al de structure B2: Influence d'une interaction entre lacunes thermiques et atomes de bore
}

\author{
A.-S. Gay, A. Fraczkiewicz, M. Biscondi
}

\section{- To cite this version:}

A.-S. Gay, A. Fraczkiewicz, M. Biscondi. Ségrégation intergranulaire du bore dans Fe-40 at\%Al de structure B2: Influence d'une interaction entre lacunes thermiques et atomes de bore. Journal de Physique IV Proceedings, 1996, 06 (C2), pp.C2-153-C2-158. 10.1051/jp4:1996221 . jpa-00254199

HAL Id: jpa-00254199

https://hal.science/jpa-00254199

Submitted on 1 Jan 1996

HAL is a multi-disciplinary open access archive for the deposit and dissemination of scientific research documents, whether they are published or not. The documents may come from teaching and research institutions in France or abroad, or from public or private research centers.
L'archive ouverte pluridisciplinaire HAL, est destinée au dépôt et à la diffusion de documents scientifiques de niveau recherche, publiés ou non, émanant des établissements d'enseignement et de recherche français ou étrangers, des laboratoires publics ou privés. 


\title{
Ségrégation intergranulaire du bore dans $\mathrm{Fe}-40$ at\% Al de structure B2: Influence d'une interaction entre lacunes thermiques et atomes de bore
}

\author{
A.-S. Gay, A. Fraczkiewicz et M. Biscondi \\ Ecole des Mines de Saint-Etienne, CNRS, URA 1884, 158 Cours Fauriel, 42023 Saint-Etienne cedex 2, \\ France
}

\begin{abstract}
Boron segregation to grain boundaries was studied in ordered (B2) FeA1 containing 40 at $\%$ Al. It was observed that small additions of boron (200 at ppm) change the fracture mode from intergranular to mixed (transgranular and intergranular). A low level of boron (few at \%) was detected by AES in grain boundaries of this hypostoechiometric alloy. Nevertheless, the kinetic of boron segregation is rapid : even a quench from $950^{\circ} \mathrm{C}$ promotes it. As FeAl alloys are known to retain large concentrations of thermal vacancies, the possibility of an interaction between boron atoms and vacancies was investigated in order to understand the mechanism of boron segregation. Dilatometric isothermal experiments showed that vacancies eliminate more rapidly in B-doped Fe-40 at\%Al than in B-free Fe-40 at\%Al. Modelisation of the kinetics of vacancies elimination predict that complexes boron atoms - vacancies migrate more rapidly than free vacancies : the difference between their migration energies is higher than $0,4 \mathrm{eV}$. This result could justify the assumption that at least a part of boron segregation in $\mathrm{FeAl}$ is due to a non-equilibrium mechanism.
\end{abstract}

\section{RTRODUCTION}

Les alliages FeAl ordonnés de structure B2 présentent de bonnes propriétés mécaniques à haute température $\left(650^{\circ} \mathrm{C}\right)$, mais sont fragiles à température ambiante [1] : leur rupture se fait de façon intergranulaire dans toute la gamme des teneurs en aluminium comprises entre 40 et 50 at \%. Leur fragilité est imputée entre autres à la faiblesse intrinsèque des joints de grains [2]; un ajout de faibles quantités de bore (quelques centaines de ppm atomiques) peut y remédier [3]. Un effet analogue a été constaté dans l'intermétallique $\mathrm{Ni}_{3} \mathrm{Al}$ [4] et est attribué à la ségrégation intergranulaire du bore.

La présence de bore aux joints de grains de l'alliage $\mathrm{Fe}-40$ at\% $\mathrm{Al}$ a déjà été constatée par plusieurs auteurs (par exemple [5]). Par contre, ni le mécanisme élémentaire gouvernant la ségrégation (d'équilibre ou hors équilibre), ni l'origine de l'effet bénéfique du bore n'ont été identifiés.

L'étude présentée dans cet article concerne la ségrégation intergranulaire du bore dans l'alliage $\mathrm{FeAl}$ de structure B2 contenant 40 at\% Al. Tout d'abord, l'effet du bore sur le mode de rupture de FeAl est examiné et la ségrégation intergranulaire du bore en fonction des cycles thermiques appliqués au matériau est mesurée par spectrométrie d'électrons Auger. Au vu de ces mesures, l'éventualité que la ségrégation du bore résulte, au moins partiellement, d'un mécanisme hors d'équilibre a été étudiée.

D'une façon générale, deux mécanismes principaux peuvent être responsables de la ségrégation : un, dit d'« équilibre », où la force motrice vise à rétablir l'équilibre thermodynamique des atomes ségrégeants ; l'autre, dit "hors équilibre ", fondé sur linteraction entre les atomes ségrégeants et le flux de lacunes thermiques. Or, les alliages FeAl de structure B2 ont la particularité de pouvoir retenir de grandes concentrations de lacunes thermiques [6]. En exploitant cette faculté, la possibilité d'une interaction entre les lacunes thermiques migrant vers les interfaces (joints de grains, ...) et les atomes de bore ségrégeants est étudiée. 


\section{TECHNIQUES EXPERIMENTALES}

Un alliage $\mathrm{FeAl}$ contenant 40 at\% $\mathrm{Al}$ dopé ou non par $200 \mathrm{ppm}$ atomiques de bore a été élaboré par fusion sous atmosphère d'argon en nacelle. Des échantillons parallélépipédiques $\left(1 \times 1 \times 15 \mathrm{~mm}^{3}\right)$ destinés aux mesures par spectrométrie d'électrons Auger ont subi les traitements thermiques suivants : une trempe à l'air à la température ambiante après un recuit à $950^{\circ} \mathrm{C}$ pendant $1 \mathrm{~h}$ pour retenir une concentration élevée de lacunes thermiques, suivie ou non d'un maintien à $400^{\circ} \mathrm{C}$ pendant $24 \mathrm{~h}$ pour éliminer les lacunes thermiques.

La cinétique d'élimination des lacunes thermiques a été étudiée par des essais dilatométriques isothermes à des températures voisines de $400{ }^{\circ} \mathrm{C}$ menés sur des échantillons (de dimensions $5 \times 5 \times 35 \mathrm{~mm}^{3}$ ) trempés à I'huile à partir de $950^{\circ} \mathrm{C}$.

$\mathrm{Vu}$ que la rupture de $\mathrm{Fe}-40$ at\% $\mathrm{Al}$ dopé au bore se fait de manière mixte (clivage et intergranulaire), préalablement à l'étude en spectrométrie d'électrons Auger, un chargement cathodique en hydrogène est effectué pour promouvoir la rupture intergranulaire des échantillons. Les conditions opératoires sont les suivantes : électrolyte : $1 \mathrm{~N} \mathrm{H}_{2} \mathrm{SO}_{4}+50 \mathrm{mg} / 1 \mathrm{As}_{2} \mathrm{O}_{3}$, potentiel : $-900 \mathrm{mV}$, densité de courant : $-0,2 \mathrm{~A} / \mathrm{cm}^{2}$, durée : $24 \mathrm{~h}$. Grâce au chargement cathodique en hydrogène, le pourcentage de rupture intergranulaire a sensiblement augmenté par rapport aux échantillons non chargés.

La ségrégation intergranulaire du bore a été étudiée par spectrométrie d'électrons Auger sur des surfaces de rupture produites in situ. L'acquisition des spectres est faite en mode direct. L'analyse quantitative est effectuée en mesurant l'aire sous les pics Auger du fer $(48 \mathrm{eV})$, de l'aluminium $(64 \mathrm{eV})$ et du bore $(170 \mathrm{eV})$. L'utilisation d'étalons internes (Fe-40 at\% $\mathrm{Al}$ et $\mathrm{Fe}-46$ at $\% \mathrm{Al}$ et $\mathrm{Fe}-17$ at $\% \mathrm{~B}$ ) a pernuis le calcul des concentrations intergranulaires [7]. La nature des faciès de rupture des échantillons Auger a été contrôlée par microscopie électronique à balayage.

\section{EFFET DU BORE SUR LE MODE DE RUPTURE. MESURE DE LA SEGREGATION INTERGRANULAIRE}

A température ambiante, la rupture de $\mathrm{Fe}-40$ at $\%$ Al non dopé au bore se fait de manière intergranulaire fragile (figure 1.a). L'analyse par spectrométrie d'électrons Auger ne révèle aucune ségrégation intergranulaire, confirmant l'origine intrinsèque de cette rupture fragile. Par contre, si le même alliage est dopé au bore ( $200 \mathrm{ppm}$ atomiques), la rupture devient mixte : intergranulaire et transgranulaire par clivage (figure 1.b). La proportion entre ces deux modes de rupture varie peu, que la trempe à partir de $950{ }^{\circ} \mathrm{C}$ ait été suivie ou non d'un maintien à $400^{\circ} \mathrm{C}$. En particulier, une trempe après le recuit à $950{ }^{\circ} \mathrm{C}$ suffit pour observer un renforcement des joints de grains.

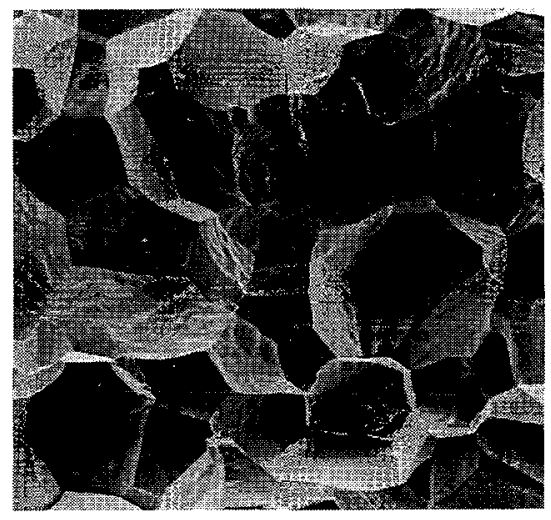

Figure 1.a : rupture intergranulaire de $\mathrm{Fe}-40$ at $\% \mathrm{Al}$ pur.

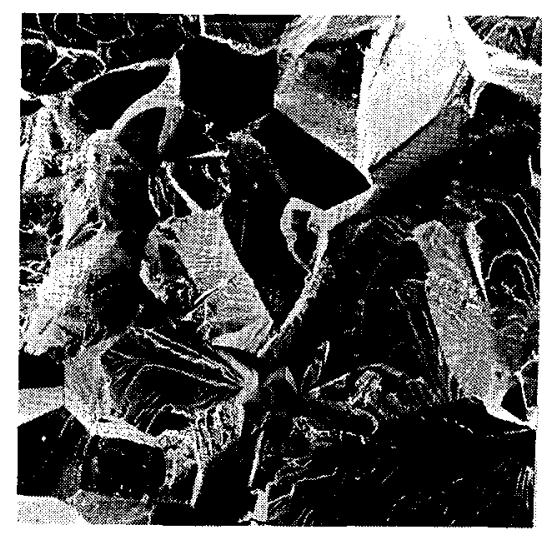

Figure 1.b : npture mixte de Fe-40 at\% Al dopé au bore 
Une faible ségrégation intergranulaire en bore a été observée par spectrométrie d'électrons Auger sur des surfaces intergranulaires de l'alliage $\mathrm{Fe}-40$ at $\% \mathrm{Al}$. La teneur en bore détectée daus les échantillons préalablement chargés en hydrogène (et qui présentent une plus grande proportion de rupture intergranulaire) est légèrement supérieure à celle d'échantillons non fragilisés. Cependant, son niveau reste faible, il ne dépasse pas 3 at $\%$ ou 7 at $\%$ selon que l'on considère que l'échantillon est homogène (à l'échelle du libre parcours moyen des électrons Auger) ou que la ségrégation a beu sur une couche. Ce niveau de ségrégation correspond à un taux d'enrichissement inférieur à 200 , valeur similaire à celle concernant la ségrégation intergranulaire du bore dans $\mathrm{Ni}_{3} \mathrm{Al}$ [8]. D'autre part, il est probable que le taux de ségrégation du bore dans les joints de grains davantage renforcés, et donc non rompus, soit supérieur. Par ailleurs, la teneur intergranulaire en bore est relativement peu sensible aux traitements thermiques appliqués au cours de l'étude au matériau.

\section{EFFET D'INTERACTION ENTRE LACUNES THERMIQUES ET ATOMES DE BORE}

Dans l'objectif de comprendre la ségrégation intergranulaire du bore, l'hypothèse d'une interaction entre lacunes thermiques et atomes de bore a été testée. Un tel phénomène a déjà été observé dans des aciers [9], où des interstitiels peuvent ètre entraînés par le flux de lacunes en migration et ségréger ainsi aux joints de grains. Cette interaction peut être mise en évidence en comparant les cinétiques d'élimination des lacunes dans des alliages dopés ou non au bore.

\subsection{Mise en évidence des cinétiques d'élimination des lacunes par dilatométrie}

La capacité des alliages $\mathrm{FeAl}$ de structure $\mathrm{B} 2$ à retenir, après trempe, une grande quantité de lacunes thermiques résulte des valeurs relativement faible de l'énergie de formation des lacunes et relativement élevée de leur énergie de migration (respectivement $0,8 \mathrm{eV}$ et $1,5 \mathrm{eV}$ pour l'alliage $\mathrm{Fe}-40$ at $\%$ Al dans les conditions thermiques décrites dans [6]). Pendant un recuit, les lacunes retenues en excès s'éliminent dans des puits tels que la surface libre ou les joints de grains.

Des mesures de dilatométrie permettent de suivre la cinétique d'élimination des lacunes thermiques. En effet, un échantillon contenant des lacunes en excès subit un retrait pendant un recuit ultérieur, lié à la concentration en lacunes éliminées par la relation: $\frac{\mathrm{dN}}{\mathrm{N}} \approx 3 \frac{\mathrm{dl}}{1}$, où $\mathrm{dN} / \mathrm{N}$ est la teneur relative en lacunes et d1/1 la contraction relative de l'échantillon. Par contre, de telles mesures ne rendent compte que d'un comportement macroscopique, qui est la somme des contributions de différents types de lacunes (situées sur les sites $\mathrm{Fe}$ ou $\mathrm{Al}$ ) ou d'amas de lacunes (de tailles diverses).

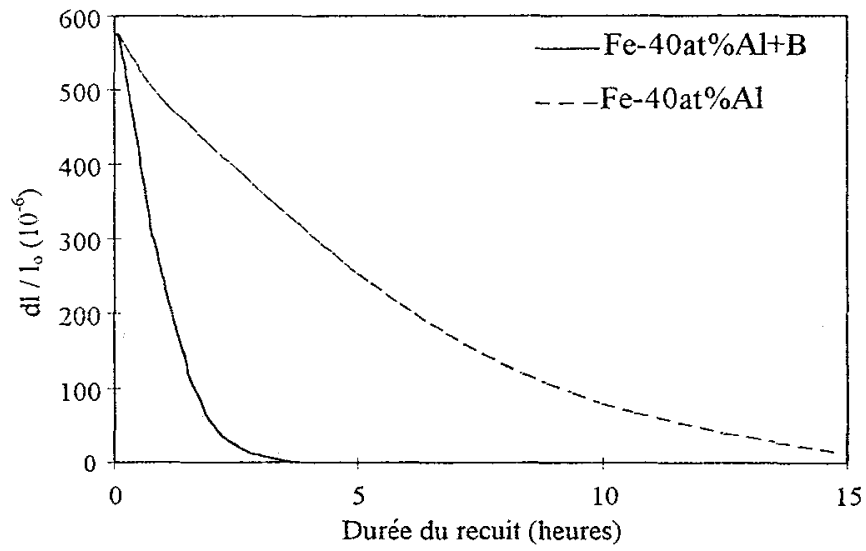

Figure 2 : Courbes dilatométriques traduisant l'élimination des lacunes.
Deux alliages $\mathrm{Fe}-40$ at $\%$ Al dopés ou non en bore ont subi des essais dilatométriques en condition isotherme après une trempe depuis $950^{\circ} \mathrm{C}$. A titre d'exemple, la figure 2 présente la cinétique d'élimination des lacunes à $395^{\circ} \mathrm{C}$.

Le retrait des échantillons dû à l'élimination des lacunes est pratiquement le mème dans les deux cas et correspond à une teneur en lacunes de $0,2 \%$. Par contre, la différence dans la cinétique d'élimination des lacunes est importante : le temps nécessaire pour atteindre la teneur d'équilibre est presque six fois supérieur dans l'alliage pur que dans l'alliage dopé. 


\subsection{Energies de migration}

\subsection{Présentation du modèle et hypothèses}

Afin de quantifier la différence de cinétique d'élimination des lacunes, un modèle [10] fondé sur des réactions chimiques entre les espèces a été utilisé pour calculer l'énergie de migration des lacunes dans les deux alliages. Les deux cas suivants ont été considérés :

- dans l'alliage sans bore, les lacunes migrent seules vers les puits (énergie de migration $\mathrm{E}_{\mathrm{m}}$ );

- daus l'alliage dopé au bore, tous les atomes de bore se lient avec les lacunes pour former des complexes caractérisés par une énergie de liaison $E_{1}$ (négative) et une énergie de migration vers les puits $E_{m}^{c}$.

Ce modèle propose une représentation macroscopique de l'interaction possible entre lacumes et atomes de bore. En particulier, la nature des puits n'est pas précisée, mais il est raisonnable d'admettre qu'il s'agit des joints de grains. De même, ui la nature de l'interaction entre lacunes et atomes de bore, ni le type de lacunes concerné ne peuvent être atteints par cette description globale du phénomène. La mise en équation du modèle est décrite en annexe.

\subsubsection{Evaluation des énergies de migration à partir des courbes expérimentales}

Pour l'alliage pur, la fraction atomique de lacunes retenues en excès $N$ varie en fonction de la durée du recuit d'élimination selon la loi (anmexe): $N=N_{0} \exp \left(-K_{\text {pur }} \cdot t\right)$, avec $K_{\text {pur }} \alpha \exp \left(-\frac{E_{m}}{k T}\right)$.

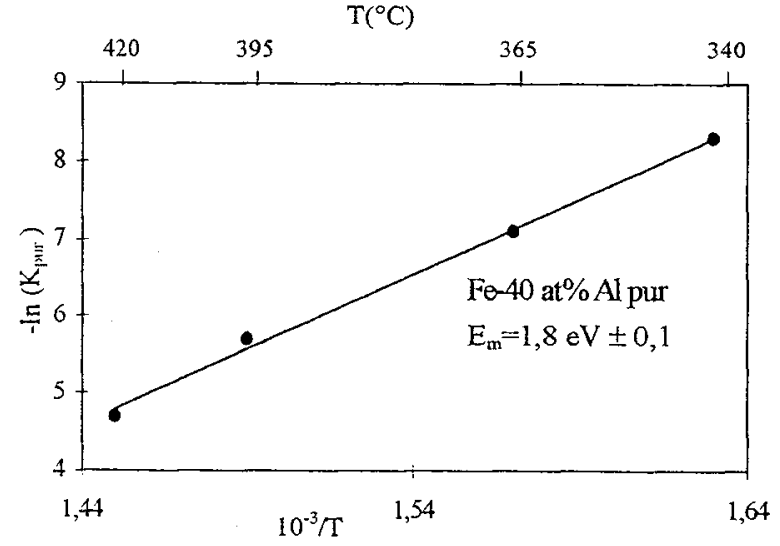

La pente des courbes dilatométriques à différentes températures tracées en coordonnées semi-logarithmiques $(t, \ln N)$ fournit les valeurs $K_{\text {pur }}(T)$. L'énergie de migration des lacunes se déduit de la pente de la droite $\left(1 / \mathrm{T}, \ln \mathrm{K}_{\text {pur }}\right)$ (voir figure 3 ). L'énergie de migration des lacunes dans l'alliage $\mathrm{Fe}-40$ at $\% \mathrm{Al}$ pur trempé depuis $950^{\circ} \mathrm{C}$ est égale à $1,8( \pm 0,1) \mathrm{eV}$. Cette valeur de l'énergie de migration est cohérente avec des résultats présentés dans la littérature [6].

Figure 3 :Calcul de l'énergie de migration dans FeAl pur.

Pour l'alliage dopé au bore, le calcul développé en annexe (relation 3) démontre la loi suivante $\mathrm{N} \propto \exp \left(-\mathrm{K}_{\text {dopé }} \cdot \mathrm{t}\right), \quad$ avec $\quad \mathrm{K}_{\text {dopé }} \approx \mathrm{K}_{\mathrm{pur}}\left(1+\beta \cdot \exp \left(\frac{\mathrm{E}_{\mathrm{m}}+\mathrm{E}_{1}-\mathrm{E}_{\mathrm{m}}^{\mathrm{c}}}{\mathrm{kT}}\right)\right)$. La pente des courbes

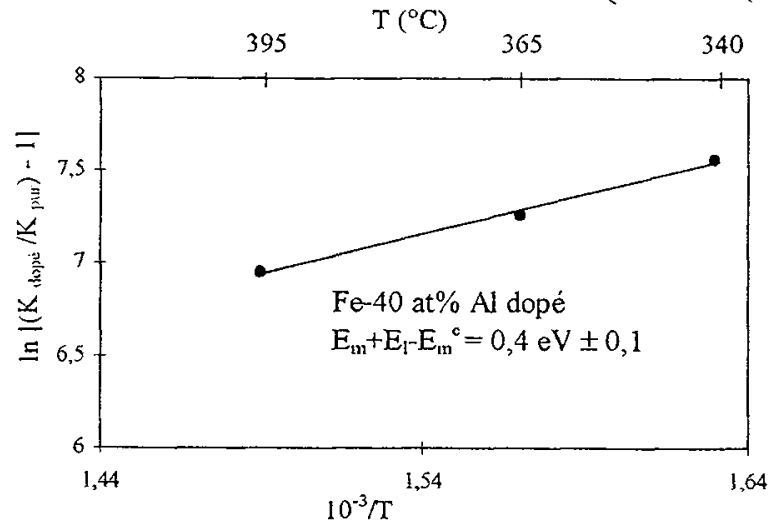

dilatométriques à différentes températures tracées en coordonnées semi-logarithmiques $(t, \ln N)$ foumit les valeurs $K_{\text {dopi }}(T)$ et la pente de la droite $\left(1 / T, \ln \left(\mathrm{K}_{\text {dopi }} / \mathrm{K}_{\text {pur }}-1\right)\right)$ (voir figure 4) fournit la valeur de $E_{m}+E_{1}-E_{m}^{c}$. Dans le cas étudié, elle vaut $0,4( \pm 0,1) \mathrm{eV}$. Counaissant l'énergie de migration des lacunes et sachant que $E_{l}$ est négative, l'énergie de migration des complexes a pu être déterminée inférieure à $1,4 \mathrm{eV}$.

Figure 4 : Calcul de l'énergie de migration dans $\mathrm{FeAl}$ dopé. 
Cette borne supérieure est inférieure à l'énergie de migration des lacunes seules : les complexes lacunesatomes de bore migrent donc plus facilement que les lacunes seules. Le fait que la formation de complexes favorise la migration des lacunes remplit une condition nécessaire pour la ségrégation hors équilibre d'atomes de soluté. Ce résultat permet d'avancer qu'au moins une partie de la ségrégation intergranulaire du bore peut être gouvernée par un mécanisme hors d'équilibre.

\section{CONCLUSION}

- Il a été confirmé que, dans l'alliage $\mathrm{FeAl}$ contenant 40 at\% $\mathrm{Al}$, l'ajout de $200 \mathrm{ppm}$ atomiques de bore change le mode de rupture d'intergranulaire à mixte (transgranulaire par clivage et intergranulaire).

- La ségrégation intergranulaire en bore a été mise en évidence par spectrométrie d'électrons Auger ; ceci a été facilité par un chargement cathodique en hydrogène, qui permet de fragiliser intergranulairement les échantillons. La teneur intergranulaire en bore est faible ( 3 à 7 at \%), ce qui correspond à un taux d'enrichissement inférieur à 200 .

- La cinétique d'élimination des lacunes thermiques (visualisée par dilatométrie) est plus rapide dans l'alliage $\mathrm{Fe}-40$ at\% $\mathrm{Al}$ dopé au bore que dans l'alliage pur. La différence de cinétique traduit une interaction entre le flux de lacunes thermiques retenues dans le matériau et les atomes de bore : les lacunes et les atomes forment des complexes qui migrent plus facilement que les lacunes seules (la différence entre les énergies de migration est supérieure à $0,4 \mathrm{eV}$ ).

- Au moins une partie de la ségrégation intergranulaire du bore pourrait ainsi résulter d'un mécanisme hors d'équilibre.

\section{BIBLIOGRAPHIE}

[1] P.M. Morgand, P. Mouturat, G. Saintfort, Acta Metall., 16 (1968), 867.

[2] P. Nagpal, I. Baker, Materials Characterization, 27 (1991), 167.

[3] M.A. Crimp, K. Vedula, Mat. Sci. and Eng., 76 (1986), 193.

[4] K. Aoki, O. Izumi, Nippon Kinzoku Gakkaishi, 41 (1977), 170.

[5] C.T. Liu, E.P. George, Scripta Metall., 24 (1990), 1285.

[6] J. Rieu, C. Goux, Mém. Sci. Rev. Mét., 66 (1969), 869.

[7] M.-T. Simonetta-Perrot, C. Lorenzon, M. Biscondi, Surf. and Interf. Analysis, 21 (1994), 316.

[8] A. Choudhury, C.L. White, C.R. Brooks, Acta Metall., 40 (1992), 57.

[9] L. Karlsson, H. Norden, H. Odelius, Acta Metall., 36 (1988), 1.

[10] A.C. Damask, G.J. Dienes, Point Defects in Metals, (Gordon and Breach, New York, 1963), 88.

\section{ANNEXE}

Le modele utilisé pour calculer les énergies de migration est développé par A.C. Damask et G.J. Dienes [10]. Pour calculer l'énergie de migration des lacunes dans l'alliage Fe-40 at\% Al pur, le modèle prévoit que les lacunes retenues en sursaturation dans le matériau migrent seules vers les puits: $\mathrm{V} \stackrel{\mathrm{K}_{\text {pur }}}{\longrightarrow}$ puits, où $K_{\text {pur }}$ est une constante de vitesse de réaction.

La réaction étant supposée d'ordre 1, ce qui est en accord avec le comportement observé (cf Figure 2), la fraction atomique en lacunes en excès varie avec le temps selon la loi suivante :

$$
\mathrm{N}=\mathrm{N}_{\mathrm{o}} \exp \left(-\mathrm{K}_{\text {pur }} \mathrm{t}\right) \text {, avec } \mathrm{K}_{\mathrm{pur}}=\frac{1}{\mathbf{n} \tau_{\mathrm{o}}} \exp \left(-\frac{\mathrm{E}_{\mathrm{m}}}{\mathrm{kT}}\right)
$$

où $n$ est le nombre moyen de sauts et $\tau_{o}$ l'inverse de la fréquence de vibration vo (estimée à $10^{I 3} \sec ^{-1}$ ).

La mise en équation devient plus compliquée dans l'alliage Fe-40 at\% Al dopé au bore. Le modèle prévoit que les lacunes $(V)$ interagissent avec les atomes de bore (I) pour former des complexes (C) et les entraînent vers les puits. 
Trois réactions sont alors à prendre en compte :

- formation des complexes lacunes - atomes de bore : $\mathrm{V}+\mathrm{I} \underset{\mathrm{K}_{2}}{\stackrel{\mathrm{K}_{1}}{\rightleftarrows}} \mathrm{C}$

- migration des lacunes libres vers les puits : $\quad \mathrm{V} \stackrel{\mathrm{K}_{3}}{\longrightarrow}$ puits

- migration des complexes vers les puits: $\quad \mathrm{C} \stackrel{\mathrm{K}_{4}}{\longrightarrow}$ puits

La concentration de bore étant d'un ordre de grandeur inférieure à celle des lacunes, il a été considéré que tous les atomes de bore se retrouvent sous forme de complexes : la ségrégation des atomes de bore seuls a donc été négligée.

Les constantes de vitesse de réaction peuvent être explicitées en introduisant les énergies de migration des lacunes, des complexes et l'énergie de liaison entre les lacunes et les atomes de bore:

$$
\mathrm{K}_{1}=\alpha \mathrm{zv} \exp \left(-\frac{\mathrm{E}_{\mathrm{m}}}{\mathrm{kT}}\right) \quad \mathrm{K}_{2}=\alpha v \exp \left(-\frac{\left(\mathrm{E}_{\mathrm{m}}+\mathrm{E}_{1}\right)}{\mathrm{kT}}\right)
$$

avec $z$ : coordinence (=8 pour un cc), $\alpha$ :nombre de façons permettant à une lacune attachée à une impureté de sauter pour s'en libérer, $v$ : fréquence de vibration $\left(=10^{13} \sec ^{-1}\right)$

$$
\mathbf{K}_{3}=\frac{1}{\mathbf{n} \tau_{\mathbf{o}}} \exp \left(-\frac{\mathbf{E}_{\mathbf{m}}}{\mathrm{kT}}\right)=\mathbf{K}_{\text {pur }} \quad \mathbf{K}_{4}=\frac{1}{\mathbf{n}^{\prime} \tau_{\mathbf{o}}^{\prime}} \exp \left(-\frac{\mathbf{E}_{\mathbf{m}}^{\mathrm{c}}}{\mathrm{kT}}\right)
$$

avec n' : nombre moyen de sauts des complexes, $\tau_{0}^{\prime}$ : inverse de la fréquence de vibration.

Lorsque le régime permanent est installé, les fractions atomiques en lacunes isolées $(V)$, en complexes (C), en impuretés (I) et la fraction atomique totale en lacunes en excès $(N=C+V)$ sont lieees à la fraction atomique initiale en bore $\left(\Lambda_{0}\right)$ par les relations :

$$
\left\{\begin{array}{l}
\frac{\mathrm{dC}}{\mathrm{dt}}=-\mathrm{K}_{1} \mathrm{VI}-\mathrm{K}_{2} \mathrm{C}-\mathrm{K}_{4} \mathrm{C} \text { avec } I=I_{o}-\mathrm{C} \\
\frac{\mathrm{dV}}{\mathrm{dt}}=-\mathrm{K}_{1} \mathrm{VC}+\mathrm{K}_{1} \mathrm{VI}_{\mathrm{o}}+\mathrm{K}_{2} \mathrm{C}-\mathrm{K}_{3} \mathrm{~V}
\end{array}\right.
$$

En conséquence de (1) et (2), $\frac{\mathrm{dN}}{\mathrm{dt}}=\frac{\mathrm{d}(\mathrm{C}+\mathrm{V})}{\mathrm{dt}}=-\mathrm{K}_{3} \mathrm{~V}-\mathrm{K}_{4} \mathrm{C}$

L'équilibre local des espèces se traduit par : $\frac{\mathrm{K}_{1}}{\mathrm{~K}_{2}}=\frac{\mathrm{C}}{\mathrm{V}\left(\mathrm{I}_{\mathbf{o}}-\mathrm{C}\right)}$

En intégrant (3), compte ternu de (4) :

$\mathrm{N}=\mathrm{C}+\mathrm{V}=\mathrm{V}_{0}\left(1+\mathrm{I}_{0} \frac{\mathrm{K}_{1}}{\mathrm{~K}_{2}}\right) \exp \left(-\mathrm{K}_{\mathrm{dopé}} \cdot \mathrm{t}\right)$, où $V_{0}$ est la fraction atomique initiale en lacunes isolées.

$$
\text { avec: } K_{\text {dopé }}=\frac{K_{3} K_{2}+K_{1} K_{4} I_{0}}{K_{2}+K_{1} I_{0}}=K_{p u r} \frac{1+8 I_{0} \frac{n \tau_{0}}{n^{\prime} \tau_{0}^{\prime}} \exp \left(\frac{E_{p}+E_{1}-E_{m}^{c}}{k T}\right)}{1+8 I_{0} \exp \left(\frac{E_{1}}{k T}\right)}
$$

L'expression de $K_{\text {dope }}$ se simplifie car :

- compte tenu de l'attraction entre les lacunes et les atomes de bore, $8 \mathrm{I}_{0} \exp \left(\frac{\mathrm{E}_{1}}{\mathrm{kT}}\right)$ est négligeable ;

- on émet l'hypothèse que la fréquence de sauts des complexes est la même que celle des lacunes seules $\left(n^{\prime} \tau_{o}^{\prime}=n \tau_{o}\right)$.

$$
\text { On peut alors écrire : } \mathrm{K}_{\mathrm{dopé}} \approx \mathrm{K}_{\mathrm{pur}}\left(1+\beta \cdot \exp \left(\frac{\mathrm{E}_{\mathrm{m}}+\mathrm{E}_{1}-\mathrm{E}_{\mathrm{m}}^{\mathrm{c}}}{\mathrm{kT}}\right)\right) \text {, avec } \beta=8 I_{o}
$$

